\title{
Efficacy of memantine on neuropsychiatric symptoms associated with the severity of behavioral variant frontotemporal dementia: A six-month, open-label, self-controlled clinical trial
}

\author{
PAN LI ${ }^{1,2}$, WEI QUAN ${ }^{3-5}$, YU-YING ZHOU ${ }^{1,2}$, YAN WANG $^{1,2}$, HUI-HONG ZHANG ${ }^{1}$ and SHUAI LIU ${ }^{1}$ \\ ${ }^{1}$ Department of Neurology, Tianjin Neurological Institute; \\ ${ }^{2}$ Tianjin Key Laboratory of Cerebral Vascular and Neurodegenerative Diseases, Tianjin Huanhu Hospital, Tianjin 300060; \\ ${ }^{3}$ Department of Neurosurgery, Tianjin Medical University, General Hospital; \\ ${ }^{4}$ Key Laboratory of Post-trauma Neurorepair and Regeneration in the Central Nervous System, \\ Tianjin Neurological Institute, Ministry of Education, General Hospital; ${ }^{5}$ Tianjin Key Laboratory of Injuries, \\ Variations and Regeneration of the Nervous System, General Hospital, Tianjin 300052, P.R. China
}

Received June 11, 2015; Accepted January 29, 2016

DOI: $10.3892 /$ etm.2016.3284

\begin{abstract}
Previous studies have focused on the curative effects of memantine in patients with mild-to-moderate frontotemporal lobar degeneration (FTLD); however, its benefits in patients with moderate-to-severe FTLD have not been investigated. The present study explores the behavioral, cognitive and functional effects of memantine on behavioral variant frontotemporal dementia (bvFTD) in patients with mild and moderate-to-severe stage bvFTD. A total of 42 patients with bvFTD completed a 6-month treatment plan of $20 \mathrm{mg}$ memantine daily in an open-label, self-controlled clinical trial. Patients were divided into two groups according to their Mini-Mental State Examination (MMSE) score: Mild (score, 21-26); and moderate-to-severe (score, 4-20). Primary endpoints included Neuropsychiatric Inventory Questionnaire (NPI-Q) and Clinic Dementia Rating (CDR) scores, and secondary endpoints comprised Neuropsychiatric Inventory Caregiver Distress Scale (NPI-D), MMSE, Montreal Cognitive Assessment (MoCA), Activity of Daily Life (ADL) and Hamilton Depression Rating Scale (HAMD) scores. Memantine treatment had no effect on overall NPI-Q scores, with the exception of the agitation subdomain in all patients with bvFTD. However, patients with moderate-to-severe bvFTD exhibited a better performance than patients with mild bvFTD,
\end{abstract}

Correspondence to: Professor Yu-Ying Zhou or Dr Pan Li, Department of Neurology, Tianjin Neurosurgical Institute, Tianjin Huanhu Hospital, 122 Qi-Xiang-Tai Road, Hexi, Tianjin 300060, P.R. China

E-mail: qiying789@sina.cn

E-mail: doc_panpan@163.com

Key words: frontotemporal lobar degeneration, behavioral variant frontotemporal dementia, neuropsychiatric behavior, cognitive function, open-label study, memantine demonstrated by improved NPI-Q total scores and subscales of agitation, depression, apathy and disinhibition. In the moderate-to-severe group, CDR and HAMD scores remained stable, but MMSE, MoCA and ADL scores were reduced after 6 months of treatment. Memantine was well-tolerated in patients. In conclusion, patients with moderate-to-severe bvFTD responded significantly better to memantine in comparison to patients with mild bvFTD with regard to their neuropsychiatric scores, while memantine did not present any cognitive or functional benefits in patients with mild bvFTD. A randomized, double-blind, placebo-controlled clinical trial with a larger number of patients is required to verify these promising results for patients with moderate-to-severe bvFTD.

\section{Introduction}

Frontotemporal lobar degeneration (FTLD) is increasingly recognized as a prevalent cause of dementia, particularly in individuals $<65$ years old at the time of diagnosis (1). FTLD encompasses a variety of clinical syndromes with marked behavioral, frontal executive and language symptoms that can be differentiated into the following three clinical syndromes: Behavioral variant frontotemporal dementia (bvFTD), semantic dementia (SD) and progressive non-fluent aphasia (PNFA); these three phenotypes have characteristic clinical manifestations and different patterns of brain atrophy, and are histopathologically heterogeneous $(2,3)$. This creates challenges for the establishment of an optimal pharmacological disease management model. To date, the U.S. Food and Drug Administration (FDA) has not approved any pharmacological treatments that specifically target FTLD and alter the course of the disease (4).

Memantine is a moderate-affinity, non-competitive antagonist of the N-methyl-D-aspartate (NMDA) receptor and is an FDA-approved treatment for moderate-to-severe Alzheimer's disease (AD) (5). However, a growing body of clinical evidence suggests that the beneficial effect of memantine is not limited to AD. For example, it has beneficial effects in Parkinson's disease-related forms of dementia and other mixed causes of 
dementia (6). In addition, memantine has been demonstrated to ease or improve behavioral and cognitive manifestations of these forms of dementia, in particular various behavioral disturbances (irritability, agitation, aggression and difficulty eating) (7-9).

Previously reviewed studies have observed that NMDA receptors are upregulated in FTLD and suggest that aberrant glutamatergic neurotransmission and glutamate excitotoxicity mediating cellular dysfunction may be implicated in the pathogenesis of FTLD (10). Therefore, it is hypothesized that memantine may be useful for treating the symptoms of FTLD.

Data from a number of clinical trials using memantine to treat mild-to-moderate FTLD [Mini-Mental State Examination (MMSE) score, $\geq 15$; average Clinical Dementia Rating (CDR), <2] have been published. In a small case series, memantine was observed to have beneficial effects on the total Neuropsychiatric Inventory (NPI) score, in particular improving the apathy subscale scores, and the level of agitation and anxiety (11). In an open-label study, a transient improvement of the total NPI score was observed in patients with FTLD undergoing memantine treatment (12). In addition, it was reported in one case of FTLD that symptoms of apathy improved in response to treatment with memantine (13). Furthermore, memantine was demonstrated to elevate cortical metabolic activities in the frontal and temporal regions, and in the salience network hubs in FTLD $(14,15)$, thereby improving the quality of life of patients with FTLD and their families (12). These studies highlight the potential clinical applications of memantine in FTLD; however, a randomized controlled trial for memantine demonstrated little efficacy in the treatment of FTLD, and the results suggested that it may even hasten cognitive decline $(16,17)$. This suggests that the drug should not be prescribed to patients with FTLD.

FTLD can be misdiagnosed as a mental illness, as a number of patients with FTLD may display significant personality changes, behavioral abnormalities and language impairments in the early stages of the disease (18). It was demonstrated that patients with FTLD had significantly shorter survival and faster rates of cognitive and functional decline compared to those with Alzheimer disease (AD). The years from initial evaluation to death in FTLD patients is 4.2 years, but 6.0 years in $\mathrm{AD}$ patients $(\log$-rank test=5.17, $\mathrm{P}<0.05)(19)$. According to the survey of epidemiology, from the time of symptom onset, the mean survival in all FTLD is estimated to range from 6.6 to 11.0 years. The mean survival from time of clinical diagnosis of FTLD is estimated to range between 3 and 4 years, which means that patients with FTLD admitted to hospital will progress to a moderate or severe stage in no more than 3 and 4 years $(20,21)$. The majority of studies have focused on the effects of memantine on mild-to-moderate (MMSE score, >15) FTLD and have neglected its possible benefits for the treatment of patients in moderate-to-severe stages of FTLD (16).

One study concluded that memantine demonstrates a lack of efficacy in treating patients with FTLD and therefore suggested that the drug may not improve a patient's overall behavioral and cognitive function (17); however, the study did not closely analyze the effects of memantine on specific subsets of behavioral and neuropsychiatric symptoms or cognitive impairment. Therefore, in the present study, the efficacy, safety and tolerability of a 6-month treatment regimen of memantine was evaluated in patients with FTLD. The difference between moderate-to-severe (MMSE scores, 4-20) and mild (MMSE scores, 21-26) behavioral variant frontotemporal degeneration outpatients was evaluated, and specific subscales of behavioral and cognitive functions were analyzed.

\section{Materials and methods}

Patient selection and exclusion criteria. In this 6-month open-label clinical study, 60 outpatients from October 2008 to October 2014 were diagnosed with bvFTD according to consensus diagnostic criteria $(22,23)$. These patients were enrolled in the Cognitive Impairment Clinic (CIC) at the Neurology Department of Tianjin Huanhu Hospital (Tianjin, China). Memantine drug-naive patients were enrolled in the final trial. Patients that were prescribed oxiracetam (10 patients, $0.8 \mathrm{~g}$ thrice daily), a memory-improving agent widely used in senile dementia patients, or a cholinesterase inhibitor ( 8 patients, $5 \mathrm{mg}$ once daily) were excluded from the present study. Other exclusion criteria were as follows: i) Aphasia, severe illness and difficulty completing procedures of the study; ii) the pattern of deficits was better accounted for by other non-degenerative neurological or psychiatric disorders; iii) serious chronic conditions within the previous year; iv) magnetic resonance imaging (MRI) or computed tomography (CT) findings inconsistent with bvFTD diagnosis criteria; v) biomarker results that strongly indicated a diagnosis of $\mathrm{AD}$ or other forms of dementia; vi) acetylcholinesterase inhibitor treatment and other nootropic or antipsychotic medications taken within 4 weeks of baseline measurement recording; vii) metabolic or inflammatory brain disorders.

Patients were 53-84 years old with Montreal Cognitive Assessment (MoCA) scores between 4 and 29 at the initial screening during enrollment. All participants were required to have an MRI or brain CT scan within 1 year to confirm the bvFTD diagnosis, and a number of patients received ${ }^{18} \mathrm{~F}$-fluorodeoxyglucose $\left({ }^{18} \mathrm{~F}-\mathrm{FDG}\right)$ positron emission $(\mathrm{PET}) / \mathrm{CT}$ in order to further confirm the diagnosis.

Study design and diagnostic procedures. A total of 42 drug-naive patients were treated with $10 \mathrm{mg}$ memantine (Lundbeck, Valby, Denmark), which is the maximal FDA-approved dose for the treatment of $\mathrm{AD}$, twice daily over a 6-month period. Patients were divided into the following groups according to their MMSE score: Mild bvFTD (MMSE score, 21-26); and moderate-to-severe bvFTD (MMSE score, 4-20).

The following five parameters, including subscale scores, were evaluated as primary and secondary endpoints: General condition (CDR); cognitive function [MMSE/Montreal Cognitive Assessment (MoCA)]; neuropsychiatric behavior (NPI); emotional state [Hamilton Depression Rating Scale (HAMD)]; and activities of daily living (ADL). Diagnostic procedures included the evaluation of medical history, psychiatric and neurological examination, and laboratory screening. A confirmed diagnosis from two experienced neurologists was required to conclude the diagnosis. Physical and neurological examinations, in addition to routine laboratory screening, were performed on the first hospital visit and every 4 weeks thereafter. Primary endpoint parameters included the baseline-to-endpoint changes in the NPI Questionnaire (NPI-Q) (24) and CDR; high scores indicate greater 
Table I. Baseline demographics and clinical characteristics of mild, moderate-to-severe and combined behavioral variant frontotemporal degeneration groups.

\begin{tabular}{|c|c|c|c|c|}
\hline Variable & Mild & Moderate-to-severe & Total & P-value \\
\hline Age, years ${ }^{a}$ & $67.40 \pm 1.88$ & $68.05 \pm 1.83$ & $67.74 \pm 1.30$ & 0.807 \\
\hline Gender, male/female & $8 / 12$ & $10 / 12$ & $18 / 24$ & 0.721 \\
\hline Marriage, n (\%) & $163650.00)$ & $20(90.91)$ & $36(85.71)$ & 0.570 \\
\hline Body mass index, $\mathrm{kg} / \mathrm{m}^{2} \mathrm{a}$ & $23.45 \pm 1.04$ & $21.82 \pm 0.78$ & $22.60 \pm 0.65$ & 0.212 \\
\hline Education, years $^{\mathrm{a}}$ & $10.60 \pm 0.93$ & $8.05 \pm 0.96$ & $9.26 \pm 0.70$ & 0.064 \\
\hline Hypertension, n (\%) & $3(15.00)$ & $9(40.91)$ & $12(28.57)$ & 0.130 \\
\hline Diabetes mellitus, n (\%) & $2(10.00)$ & $3(13.64)$ & $5(11.90)$ & 1.000 \\
\hline Coronary heart disease, $\mathrm{n}(\%)$ & $2(10.00)$ & $3(13.64)$ & $5(11.90)$ & 1.000 \\
\hline Cerebral trauma, n (\%) & $0(0.00)$ & $0(0.00)$ & $0(0.00)$ & \\
\hline Smoking, n (\%) & $5(25.00)$ & $6(27.27)$ & $11(26.19)$ & 0.867 \\
\hline Drinking, n (\%) & $3(15.00)$ & $4(18.18)$ & 7 (16.67) & 1.000 \\
\hline Dementia family history, n (\%) & $0(0)$ & $5(22.73)$ & $5(11.90)$ & 0.073 \\
\hline Disease duration, years ${ }^{\mathrm{a}}$ & $2.13 \pm 0.31$ & $1.77 \pm 0.29$ & $1.94 \pm 0.21$ & 0.408 \\
\hline Patient number (completed 26 weeks) & $20(20)$ & $22\left(21^{\mathrm{b}}\right)$ & $42\left(41^{\mathrm{b}}\right)$ & \\
\hline
\end{tabular}

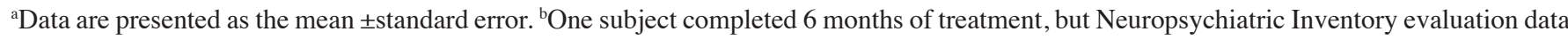
was not available; another subject succumbed to mortality during the follow-up period.

impairment. Secondary endpoint assessments included the following: Chinese version of the MoCA $(25,26)$ and MMSE $(27,28)$ scores (range, 0-30 for both; high scores indicate better cognitive function); ADL scale including two major categories, Basic Activity of Daily Life (BADL) and Instrumental Activity of Daily Life (IADL; high scores indicate greater impairment) (29,30); and the HAMD (high scores indicate greater impairment) (31).

Adverse effects. Patient safety was monitored at each hospital visit by physical and neurological examination, the recording of adverse events and performing routine laboratory analysis on the blood of the patients. Adverse events were recorded by spontaneous reporting from patients or their caregivers.

Ethical considerations. Each patient that participated in the present study provided informed consent. At each study visit, a reliable caregiver was present and supervised the administration of the patients' medication. The present study was approved by the local institutional ethical standards committee of Tianjin Huanhu Hospital (Tianjin, China) on human experimentation.

Statistical analysis. Baseline demographic qualitative variables were analyzed using the $\chi^{2}$ test, while normally distributed quantitative variables were analyzed using independent two-sample t-tests. The changes in outcome parameters between the baseline and the six-month endpoints were analyzed using the Wilcoxon-Mann-Whitney test. Statistical analyses were performed using SPSS version 21.0 (IBM SPSS, Armonk, NY, USA). Values are recorded as the mean \pm standard error or mean \pm standard deviation. All tests were two-tailed, and $\mathrm{P}<0.05$ was considered to indicate a statistically significant difference.

\section{Results}

Patient enrollment and baseline demographic data. A total of 42 patients were included in the study and 41 completed the trial. The mild and moderate-to-severe bvFTD groups comprised 20 and 22 patients, respectively. The two groups were comparable with regard to age, gender, marital status, body mass index, level of education, disease duration, accompanying diseases and family history of dementia (Table I).

Adverse events. No medically significant changes were observed during the study with regard to routine laboratory parameters or vital signs. In patients treated with memantine, the adverse events with the highest frequency were constipation (6/42), somnolence (6/42), mild headache (4/42) and dizziness (3/42). None of the patients withdrew from the study due to the experiencing of side effects.

Memantine treatment. Efficacy was determined by primary and secondary outcome measurements of the patients with bvFTD. Primary endpoints included the evaluation of CDR and NPI-Q between the baseline and final hospital visit of the memantine-treated patients with bvFTD. The mean CDR results at the final visit, in comparison with baseline values at the initial visit, were $1.77 \pm 0.12$ and $1.68 \pm 0.125$, respectively; there was no significant difference between the scores of the full cohort (Table II).

Longitudinal NPI-Q data displayed an overall change from baseline to 6 months of $3.81 \pm 2.18$ points $(Z=-0.856, P=0.392$, Table III). A detailed analysis of the NPI-Q subscale scores revealed no significant changes in the majority of subcategories such as delusions, hallucinations, depression, anxiety, elation, apathy, disinhibition, irritability, aberrant motor behavior (AMB), nighttime behavior and appetite/eating. The subscale 
Table II. Analysis of primary and secondary outcome measures between the baseline and final visit of patients with behavioral variant frontotemporal degeneration receiving memantine treatment.

\begin{tabular}{|c|c|c|c|c|}
\hline Variable & Baseline & 6 Months & Z-value & P-value \\
\hline \multicolumn{5}{|l|}{ Primary endpoints } \\
\hline Clinic dementia rating & $1.68 \pm 0.125$ & $1.77 \pm 0.12$ & -0.836 & 0.403 \\
\hline Neuropsychiatric Inventory Questionnaire & $15.76 \pm 2.250$ & $11.95 \pm 1.79$ & -0.856 & 0.392 \\
\hline \multicolumn{5}{|l|}{ Secondary endpoints } \\
\hline Neuropsychiatric Inventory Caregiver Distress & $8.49 \pm 1.420$ & $7.00 \pm 1.05$ & -0.458 & 0.647 \\
\hline Mini-Mental State Examination & $18.10 \pm 1.080$ & $16.43 \pm 1.03$ & -3.126 & $0.002^{\mathrm{a}}$ \\
\hline Montreal Cognitive Assessment, Chinese version & $12.02 \pm 1.030$ & $10.33 \pm 1.08$ & -3.026 & $0.002^{\mathrm{a}}$ \\
\hline Activity of Daily Life & $30.79 \pm 1.680$ & $35.07 \pm 1.96$ & -3.878 & $0.001^{\mathrm{a}}$ \\
\hline Basic Activity of Daily Life & $13.55 \pm 0.640$ & $14.86 \pm 0.83$ & -3.181 & $0.001^{\mathrm{a}}$ \\
\hline Instrumental Activity of Daily Living & $17.17 \pm 1.140$ & $20.26 \pm 1.23$ & -3.937 & $0.001^{\mathrm{a}}$ \\
\hline Hamilton Depression Rating Scale & $9.05 \pm 0.890$ & $8.88 \pm 0.82$ & -0.197 & 0.844 \\
\hline
\end{tabular}

Data are presented as the mean \pm standard error. ${ }^{\mathrm{a}}<0.05$ vs. baseline.

Table III. Detailed analysis of Neuropsychiatric Inventory Questionnaire subscale scores between baseline and final visit after 6 months for all patients with bvFTD receiving memantine treatment.

\begin{tabular}{|c|c|c|c|c|}
\hline Variable & Baseline & 6 Months & Z-value & P-value \\
\hline Delusions & $0.63 \pm 0.190$ & $0.59 \pm 0.19$ & -0.419 & 0.675 \\
\hline Hallucinations & $0.63 \pm 0.180$ & $0.44 \pm 0.18$ & -1.279 & 0.201 \\
\hline Agitation & $1.98 \pm 0.390$ & $1.34 \pm 0.29$ & -2.127 & $0.033^{\mathrm{a}}$ \\
\hline Depression & $1.24 \pm 0.380$ & $0.71 \pm 0.22$ & -1.638 & 0.101 \\
\hline Anxiety & $0.68 \pm 0.190$ & $0.73 \pm 0.20$ & -0.241 & 0.809 \\
\hline Elation & $0.24 \pm 0.110$ & $0.34 \pm 0.17$ & -0.447 & 0.655 \\
\hline Apathy & $2.83 \pm 0.580$ & $1.90 \pm 0.43$ & -1.432 & 0.152 \\
\hline Disinhibition & $1.24 \pm 0.450$ & $0.71 \pm 0.20$ & -0.717 & 0.473 \\
\hline Irritability & $2.02 \pm 0.380$ & $1.46 \pm 0.28$ & -1.726 & 0.084 \\
\hline Aberrant motor behavior & $1.49 \pm 0.370$ & $1.51 \pm 0.38$ & 0.000 & 1.000 \\
\hline Nighttime behavior & $1.54 \pm 0.410$ & $1.32 \pm 0.36$ & -0.460 & 0.645 \\
\hline Appetite/eating & $1.22 \pm 0.370$ & $0.85 \pm 0.26$ & -1.011 & 0.312 \\
\hline Total scores & $15.76 \pm 2.250$ & $11.95 \pm 1.79$ & -0.856 & 0.392 \\
\hline
\end{tabular}

Data are presented as the mean \pm standard error. ${ }^{\mathrm{a}}<0.05$ vs. baseline. bvFTD, behavioral variant frontotemporal dementia.

for agitation was an exception, which was significantly different between the baseline and the final visit $(\mathrm{Z}=-2.127$, $\mathrm{P}=0.033$; Table III).

Of the secondary endpoints analyzed in patients with bvFTD treated with memantine, no statistically significant differences were detected between the total NPI Caregiver Distress (NPI-D) and HAMD scores between the baseline and final visit (Table II). By contrast, significant reductions in the total score of the MMSE $(\mathrm{Z}=-3.126, \mathrm{P}=0.002)$ and MoCA, Chinese version $(Z=-3.026, P=0.002)$ were observed, and a significant increase was observed in ADL $(Z=-3.878$, $\mathrm{P}=0.001)$, BADL $(\mathrm{Z}=-3.181, \mathrm{P}=0.001)$ and $\mathrm{IADL}(\mathrm{Z}=-3.937$, $\mathrm{P}=0.001)$ between the baseline and final visit. This indicates the presence of cognitive and functional deterioration in patients with bvFTD treated with memantine.
Subgroup analysis of patients with mild $(n=20)$ and moderate-to-severe $(n=22) b v F T D$. In order to determine whether the primary endpoints were affected by disease severity, patients with mild $(n=20)$ and moderate-to-severe $(n=22)$ bvFTD were analyzed separately (Table IV). Following 6 months of memantine treatment, the subgroup of patients with moderate-to-severe bvFTD exhibited significantly improved total NPI-Q scores $(Z=-2.488, P=0.013)$, and significant improvements in the subscales of agitation $(Z=-2.058$, $\mathrm{P}=0.04)$, depression $(\mathrm{Z}=-2.511, \mathrm{P}=0.012)$, apathy $(\mathrm{Z}=-2.586$, $\mathrm{P}=0.01)$ and disinhibition $(\mathrm{Z}=-2.047, \mathrm{P}=0.041)$ compared with those at baseline. By contrast, memantine caused no significant changes in patients with mild bvFTD with regard to the total NPI-Q score $(14.50 \pm 2.82$ baseline vs. $15.70 \pm 3.00$ after 6 months, $\mathrm{P}=0.192$ ) or individual subscale scores. After 
Table IV. Detailed analysis of Neuropsychiatric Inventory Questionnaire neuropsychiatric subdomains between mild and moderate-to-severe behavioral variant frontotemporal dementia in patients receiving memantine treatment.

\begin{tabular}{|c|c|c|c|c|c|c|}
\hline \multirow[b]{2}{*}{ Variable } & \multicolumn{3}{|c|}{ Mild $(n=20)$} & \multicolumn{3}{|c|}{ Moderate-to-severe $(n=20)$} \\
\hline & Baseline & 6 Months & P-value & Baseline & 6 Months & P-value \\
\hline Delusions & $0.35 \pm 0.20$ & $0.65 \pm 0.33$ & 0.109 & $0.90 \pm 0.32$ & $0.52 \pm 0.20$ & 0.113 \\
\hline Hallucinations & $0.45 \pm 0.25$ & $0.55 \pm 0.34$ & 0.414 & $0.81 \pm 0.27$ & $0.33 \pm 0.17$ & 0.068 \\
\hline Agitation & $2.10 \pm 0.57$ & $1.40 \pm 0.34$ & 0.233 & $1.86 \pm 0.54$ & $1.29 \pm 0.48$ & $0.040^{\mathrm{a}}$ \\
\hline Depression & $1.35 \pm 0.70$ & $1.00 \pm 0.41$ & 0.595 & $1.14 \pm 0.37$ & $0.43 \pm 0.16$ & $0.012^{\mathrm{a}}$ \\
\hline Anxiety & $0.65 \pm 0.25$ & $1.20 \pm 0.37$ & 0.077 & $0.71 \pm 0.29$ & $0.29 \pm 0.12$ & 0.071 \\
\hline Elation & $0.10 \pm 0.10$ & $0.40 \pm 0.31$ & 0.317 & $0.38 \pm 0.19$ & $0.29 \pm 0.17$ & 0.317 \\
\hline Apathy & $2.50 \pm 0.69$ & $3.15 \pm 0.77$ & 0.305 & $3.14 \pm 0.93$ & $0.71 \pm 0.23$ & $0.010^{\mathrm{a}}$ \\
\hline Disinhibition & $1.15 \pm 0.70$ & $0.90 \pm 0.26$ & 0.382 & $1.33 \pm 0.57$ & $0.52 \pm 0.30$ & $0.041^{\mathrm{a}}$ \\
\hline Irritability & $2.00 \pm 0.55$ & $1.95 \pm 0.49$ & 0.862 & $2.05 \pm 0.55$ & $1.00 \pm 0.24$ & 0.073 \\
\hline Aberrant motor behavior & $1.50 \pm 0.61$ & $1.80 \pm 0.63$ & 0.180 & $1.48 \pm 0.43$ & $1.24 \pm 0.44$ & 0.354 \\
\hline Nighttime behavior & $1.60 \pm 0.52$ & $1.80 \pm 0.65$ & 0.684 & $1.48 \pm 0.64$ & $0.86 \pm 0.33$ & 0.498 \\
\hline Appetite/Eating & $0.75 \pm 0.33$ & $0.90 \pm 0.39$ & 0.655 & $1.67 \pm 0.65$ & $0.81 \pm 0.34$ & 0.173 \\
\hline Total scores & $14.50 \pm 2.82$ & $15.70 \pm 3.00$ & 0.192 & $16.95 \pm 3.52$ & $8.38 \pm 1.76$ & $0.013^{\mathrm{a}}$ \\
\hline
\end{tabular}

Data are presented as the mean \pm standard deviation; ${ }^{a} \mathrm{P}<0.05$ vs. baseline.

Table V. Changes in Neuropsychiatric Inventory Questionnaire scores in memantine-treated patients with mild and moderate-to-severe behavioral variant frontotemporal dementia.

\begin{tabular}{lcc}
\hline Observation & Mild $^{\mathrm{a}, \mathrm{b}}$ & Moderate-to-Severe $^{\mathrm{a}, \mathrm{b}}$ \\
\hline Improved & 5 & 12 \\
No change & 3 & 4 \\
Worsened & 12 & 4 \\
\hline
\end{tabular}

${ }^{\mathrm{a}} \chi^{2}$-value $=7.131,{ }^{\mathrm{b} P}$-value $=0.028$.

6 months, the comparison of NPI-Q scores between groups demonstrated that 5 patients improved, 3 patients remained stable and 12 patients worsened in the mild bvFTD group; whereas 12 patients improved, 4 patients remained stable and 4 patients worsened in the moderate-to-severe bvFTD group (Table V). The outcome proportions of the patients, on the basis of improved, unchanged or worsened NPI-Q scores, was found to differ significantly between the mild and moderate-to-severe bvFTD groups ( $\mathrm{P}=0.028$; Fig. 1).

\section{Discussion}

The present study compared the changes in cognitive function, and neuropsychiatric and behavioral symptoms, in patients with mild and moderate-to-severe bvFTD using standardized tests after 6 months of memantine treatment. Analysis of the combined cohort of 42 patients with bvFTD demonstrated that cognitive performance worsened, indicated by changes in MoCA and MMSE scores, and ability to perform activities in daily living deteriorated, indicated by changes in ADL, during the 6-month period of memantine treatment. Although

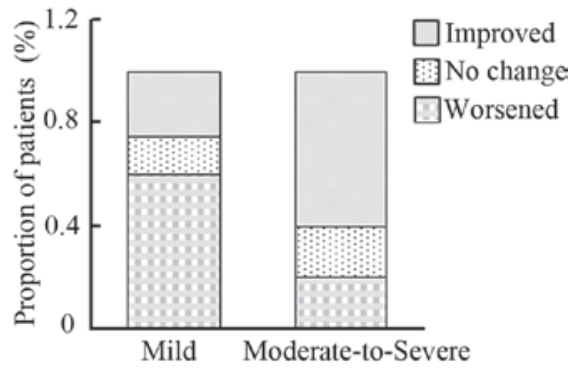

Figure 1. Neuropsychiatric Inventory Questionnaire (NPI-Q) values obtained at the final visit following 6 months of memantine treatment. In the mild behavioral variant frontotemporal degeneration (bvFTLD) group, the NPI scores worsened in 12 patients, remained stable in 3 patients and improved in 5 patients; in the moderate-to-severe bvFTD group, 4 patients worsened, 4 patients remained stable and 12 patients improved. $(\mathrm{P}=0.028)$. The P-value was used to analyze the proportion of patients that have shown improvement, no change and worsening of symptoms between the mild and moderate-to-severe groups.

memantine had no beneficial effects on the total or subscale NPI-Q scores of the combined cohort of 42 patients with bvFTD, the moderate-to-severe patient subgroup performed significantly better than the mild patient group in their response to memantine treatment. This was demonstrated by changes to the total NPI-Q score, and subscale scores for agitation, depression, apathy and disinhibition. Memantine appeared to be safe and well-tolerated, and no patient withdrew from the trial as a result of adverse reactions.

FTLD is a clinically, anatomically and histopathologically heterogeneous disorder (3), making it difficult to design a therapeutic trial that could accommodate all possible therapeutic outcome measures. This, therefore, creates particular challenges for determining an optimal management model with a single pharmacological agent. Although memantine is an FDA-approved AD medication, it is not indicated for FTLD treatment. Furthermore, 
a recent randomized controlled trial discouraged the prescription of memantine to FTLD patients $(16,17)$. Despite this, the evidence-based clinical practices guidelines, clinical experience indicates that a high percentage of patients with FTLD are treated with AChEI, memantine or both. A cross-sectional design using 1,092 cases with AD and 64 cases with FTLD were registered by the Registry of Dementias of Girona. Memantine was used by $17.2 \%$ and $10.9 \%$ of patients with AD and FTLD, respectively. There is a discrepancy regarding clinical practice and the recommendations based upon clinical evidence (32).

The aggregate analysis of neuropsychological, cognitive and functional performance across a cohort of patients with bvFTD in the present study indicated that treatment with memantine worsened a patient's cognitive function and ability to perform activities in daily living, and this is consistent with a previously reported randomized controlled clinical trial (16). Notably, each enrolled subject in the present study had significant neuropsychiatric disturbances, as assessed by NPI-Q and NPI-D scores. The high prevalence of neuropsychiatric symptoms in patients with bvFTD has been previously reported in other community-based studies $(33,34)$. Patients with moderate-to-severe bvFTD had a higher NPI-Q total score than patients with mild bvFTD. In the current study, it was observed that behavioral disturbances improved markedly in patients with moderate-to-severe bvFTD, in comparison with patients with mild bvFTD, after 6 months of memantine treatment. Detailed analysis of behavioral subscales demonstrated that the most notable improvements were observed in agitation, depression, apathy and disinhibition. Despite the relatively small sample size included in the present study, this is a potentially important finding.

Different responses to memantine treatment in patients with mild and moderate-to-severe bvFTD may be attributed to the heterogeneous profile of preserved and/or impaired brain regions, or selective functional systems in different stages of the disease. Neuropathological and imaging studies of early FTLD suggest that the disease begins in the anterior cingulate cortex (ACC) and frontoinsula (FI) before spreading throughout a circumscribed network of brain regions $(35,36)$. ACC and FI are the exclusive location of the von Economo neuron (VEN) that is specifically and selectively attacked in the early stages of bvFTD; these are implicated in several neuropsychiatric illnesses, in particular in disturbances of empathy, social awareness and self-control $(37,38)$. In addition, the VEN somatodendritic compartment appears to primarily express dopamine (D3), serotonin (5HT-1b, 2b) and vasopressin (1a) receptors, but does not respond to glutamic acid (39). VEN damage in early-stage bvFTD may explain why selective serotonin reuptake inhibitors, such as paroxetine (40) or 5-hydroxytryptamine 2A (5-HT2A) antagonists (41), but not NMDA receptor antagonists, have modest benefits in improving the behavioral symptoms of patients with mild FTLD.

In more advanced stages of bvFTD, the loss of frontal and temporal neurons becomes more severe with greater deep layer involvement as the disease spreads throughout the white matter to more posterior regions and the subcortex (42). Moreover, presynaptic proteins such as $\alpha$-synuclein and neurofilament proteins would be lost during severe cases of bvFTD; these two proteins are relatively preserved in a number of mild cases of bvFTD (43). A previous study demonstrated that memantine treatment can stimulate dendritic spine maturation and promote excitatory synapse formation, restoring excitatory synapses to a normal range in mice with fragile X syndrome (44). Based on these findings, it can be suggested that memantine exerts its therapeutic capacity by alleviating behavioral disturbances through promoting synapse formation and modulating glutamate neuronal excitability in later stages of bvFTD.

Chow et al demonstrated using ${ }^{18} \mathrm{~F}-\mathrm{FDG}$ PET analysis that patients with mild FTLD (mean CDR score, 1.35) who were treated with memantine displayed increased cortical metabolic activities in the frontal and temporal brain regions, and in the salience network hubs, although no improvements in clinical symptoms were detected $(14,15)$. This suggests that the curative effect of memantine is too weak to be detected at a heterogeneous clinical symptom level; however, the results demonstrated that memantine induces metabolic changes in patients with early stage FTLD. It remains to be tested whether pharmaceutical effects on cortical metabolic activities would lead to clinical improvements as the disease progresses from mild to moderate-to-severe stage bvFTD.

In conclusion, to the best of our knowledge, the present study is the first to evaluate the effect of memantine on detailed longitudinal changes assessed by behavioral, cognitive and functional scales in patients with mild and moderate-to-severe bvFTD. The results demonstrate that memantine improves neuropsychiatric symptoms observed in patients with moderate-to-severe bvFTD, in particular the symptoms of agitation, depression, apathy and disinhibition. However, the current data must be interpreted carefully due to the relatively small number of patients with FTLD involved in the study. In the future, larger-scale, randomized, controlled studies with hierarchical design for subtype and severity of dementia are required in order to further evaluate the efficacy of memantine in patients with moderate-to-severe bvFTD.

\section{Acknowledgements}

The present study was supported by grants from the Tianjin Health Bureau of Science and Technology Research Foundation (no. 2013KG121), the Tianjin Science and Technology Plan Foundation (no. 13ZCZDSY01600), the Special Fund of National Clinical and Medical Research (no. L2014071) and the Youth Fund of National Nature Science Foundation of China (no. 81301629).

\section{References}

1. Baborie A, Griffiths TD, Jaros E, McKeith IG, Burn DJ, Richardson A, Ferrari R, Moreno J, Momeni P, Duplessis D, et al: Pathological correlates of frontotemporal lobar degeneration in the elderly. Acta Neuropathol 121: 365-371, 2011

2. Seilhean D, Bielle F, Plu I and Duyckaerts C: Frontotemporal lobar degeneration: Diversity of FTLD lesions. Rev Neurol (Paris) 169: 786-792, 2013.

3. Shinagawa S: Phenotypic variety in the presentation of frontotemporal lobar degeneration. Int Rev Psychiatry 25: 138-144, 2013.

4. Kaye ED, Petrovic-Poljak A, Verhoeff NP and Freedman M: Frontotemporal dementia and pharmacologic interventions. J Neuropsychiatry Clin Neurosci 22: 19-29, 2010.

5. Danysz W and Parsons CG: Alzheimer's disease, $\beta$-amyloid, glutamate, NMDA receptors and memantine - searching for the connections. Br J Pharmacol 167: 324-352, 2012.

6. Kalia LV, Kalia SK and Salter MW: NMDA receptors in clinical neurology: Excitatory times ahead. Lancet Neurol 7: 742-755, 2008. 
7. Cummings JL, Schneider E, Tariot PN and Graham SM Memantine MEM-MD-SG-02 Study group: Behavioral effects of memantine in Alzheimer disease patients receiving donepezil treatment. Neurology 67: 57-63, 2006.

8. Peng D, Yuan X and Zhu R: Memantine hydrochloride in the treatment of dementia subtypes. J Clin Neurosci 20: 1482-1485, 2013.

9. Stubendorff K, Larsson V, Ballard C, Minthon L, Aarsland D and Londos E: Treatment effect of memantine on survival in dementia with Lewy bodies and Parkinson's disease with dementia: A prospective study. BMJ Open 4: e005158, 2014.

10. Lipton SA: Paradigm shift in neuroprotection by NMDA receptor blockade: Memantine and beyond. Nat Rev Drug Discov 5: 160-170, 2006

11. Swanberg MM: Memantine for behavioral disturbances in frontotemporal dementia: A case series. Alzheimer Dis Assoc Disord 21: 164-166, 2007.

12. Boxer AL, Lipton AM, Womack K, Merrilees J, Neuhaus J, Pavlic D, Gandhi A, Red D, Martin-Cook K, Svetlik D and Miller BL: An open-label study of memantine treatment in 3 subtypes of frontotemporal lobar degeneration. Alzheimer Dis Assoc Disord 23: 211-217, 2009.

13. Links KA, Black SE, Graff-Guerrero A, Wilson AA, Houle S, Pollock BG and Chow TW: A case of apathy due to frontotemporal dementia responsive to memantine. Neurocase 19 256-261, 2013.

14. Chow TW, Graff-Guerrero A, Verhoeff NP, Binns MA, Tang-Wai DF, Freedman M, Masellis M, Black SE, Wilson AA, Houle S and Pollock BG: Open-label study of the short-term effects of memantine on FDG-PET in frontotemporal dementia. Neuropsychiatr Dis Treat 7: 415-424, 2011.

15. Chow TW, Fam D, Graff-Guerrero A, Verhoeff NP, Tang-Wai DF Masellis M, Black SE, Wilson AA, Houle S and Pollock BG: Fluorodeoxyglucose positron emission tomography in semantic dementia after 6 months of memantine: An open-label pilot study. Int J Geriatr Psychiatry 28: 319-325, 2013.

16. Boxer AL, Knopman DS, Kaufer DI, Grossman M, Onyike C Graf-Radford N, Mendez M, Kerwin D, Lerner A, Wu CK et al: Memantine in patients with frontotemporal lobar degeneration A multicentre, randomised, double-blind, placebo-controlled trial. Lancet Neurol 12: 149-156, 2013.

17. Hodges JR: Hope abandoned: Memantine therapy in frontotemporal dementia. Lancet Neurol 12: 121-123, 2013

18. Bird T, Knopman D, VanSwieten J, Rosso S, Feldman H, Tanabe H, Graff-Raford N, Geschwind D, Verpillat P and Hutton M: Epidemiology and genetics of frontotemporal dementia/Pick's disease. Ann Neurol 54 (Suppl 5): S29-S31, 2003

19. Rascovsky K, Salmon DP, Lipton AM, Leverenz JB, DeCarli C, Jagust WJ, Clark CM, Mendez MF, Tang-Wai DF, Graff-Radford NR and Galasko D. Rate of progression differs in frontotemporal dementia and Alzheimer disease. Neurology 65 397-403, 2005

20. Boxer AL, Gold M, Huey E, Hu WT, Rosen H, Kramer J, Gao FB, Burton EA, Chow T, Kao A, Leavitt BR, et al. The advantages of frontotemporal degeneration drug development (part 2 of frontotemporal degeneration: the next therapeutic frontier). Alzheimers Dement: Oct 10, 2012 (Epub ahead of print) doi:10.1016/j. jalz.2012.03.003

21. Riedl L, Mackenzie IR, Förstl H, Kurz A and Diehl-Schmid J. Frontotemporal lobar degeneration: current perspectives. Neuropsychiatr Dis Treat: Feb 13, 2014. (Epub ahead of print) doi:10.2147/NDT.S38706.

22. Neary D, Snowden JS, Gustafson L, Passant U, Stuss D, Black S, Freedman M, Kertesz A, Robert PH, Albert M, et al Frontotemporal lobar degeneration: A consensus on clinical diagnostic criteria. Neurology 51: 1546-1554, 1998.

23. Rascovsky K, Hodges JR, Knopman D, Mendez MF Kramer JH, Neuhaus J, van Swieten JC, Seelaar H, Dopper EG, Onyike CU, et al: Sensitivity of revised diagnostic criteria for the behavioural variant of frontotemporal dementia. Brain 134 (Pt 9): 2456-2477, 2011

24. Wang T, Xiao S, Li X, Wang H, Liu Y, Su N and Fang Y: Reliability and validity of the Chinese version of the neuropsychiatric inventory in mainland China. Int J Geriatr Psychiatry 27 539-544, 2012

25. Freitas S, Simões MR, Alves L, Duro D and Santana I: Montreal Cognitive Assessment (MoCA): Validation study for frontotemporal dementia. J Geriatr Psychiatry Neurol 25: 146-154, 2012 .
26. Hu JB, Zhou WH, Hu SH, Huang ML, Wei N, Qi HL, Huang JW and $\mathrm{Xu}$ Y: Cross-cultural difference and validation of the Chinese version of Montreal Cognitive Assessment in older adults residing in Eastern China: Preliminary findings. Arch Gerontol Geriatr 56: 38-43, 2013.

27. Dong Y, Yean Lee W, Hilal S, Saini M, Wong TY, Chen CL, Venketasubramanian $\mathrm{N}$ and Ikram MK: Comparison of the Montreal Cognitive Assessment and the Mini-Mental State Examination in detecting multi-domain mild cognitive impairment in a Chinese sub-sample drawn from a population-based study. Int Psychogeriatr 25: 1831-1838, 2013.

28. Katzman R, Zhang MY, Ouang-Ya-Qu, Wang ZY, Liu WT, Yu E, Wong SC, Salmon DP and Grant I: A Chinese version of the Mini-Mental State Examination; impact of illiteracy in a Shanghai dementia survey. J Clin Epidemiol 41: 971-978, 1988.

29. Wang D, Zheng J, Kurosawa M, Inaba Y and Kato N: Changes in activities of daily living (ADL) among elderly Chinese by marital status, living arrangement, and availability of healthcare over a 3-year period. Environ Health Prev Med 14: 128-141, 2009.

30. Zhang M, Zhu Z and Chen P, Community investigation of the activities of daily living (ADL) and medical conditions of the elderly in Shanghai. Zhonghua Yi Xue Za Zhi 78: 124-127, 1998 (In Chinese)

31. Zheng YP, Zhao JP, Phillips M, Liu JB, Cai MF, Sun SQ and Huang MF: Validity and reliability of the Chinese Hamilton Depression Rating Scale. Br J Psychiatry 152: 660-664, 1988.

32. López-Pousa S, Calvó-Perxas L, Lejarreta S, Cullell M, Meléndez R, Hernández E, Bisbe J, Perkal H, Manzano A Roig AM, Turró-Garriga O, Vilalta-Franch J, Garre-Olmo J; Registry of Dementias of Girona Study Group (ReDeGi Study Group): Use of antidementia drugs in frontotemporal lobar degeneration. Am J Alzheimers Dis Other Demen 27: 260-266, 2012.

33. Srikanth S, Nagaraja AV and Ratnavalli E: Neuropsychiatric symptoms in dementia-frequency, relationship to dementia severity and comparison in Alzheimer's disease, vascular dementia and frontotemporal dementia. J Neurol Sci 236: 43-48, 2005.

34. Quaranta D, Marra C, Rossi C, Gainotti G and Masullo C: Different apathy profile in behavioral variant of frontotemporal dementia and Alzheimer's disease: A preliminary investigation. Curr Gerontol Geriatr Res 2012: 719250, 2012.

35. Broe M, Hodges JR, Schofield E, Shepherd CE, Kril JJ and Halliday GM: Staging disease severity in pathologically confirmed cases of frontotemporal dementia. Neurology 60 : 1005-1011, 2003.

36. Seeley WW: Selective functional, regional, and neuronal vulnerability in frontotemporal dementia. Curr Opin Neurol 21: 701-707, 2008.

37. Allman JM, Tetreault NA, Hakeem AY, Manaye KF, Semendeferi K, Erwin JM, Park S, Goubert V and Hof PR: The von Economo neurons in frontoinsular and anterior cingulate cortex in great apes and humans. Brain Struct Funct 214: 495-517, 2010.

38. Kim EJ, Sidhu M, Gaus SE, Huang EJ, Hof PR, Miller BL, DeArmond SJ and Seeley WW: Selective frontoinsular von Economo neuron and fork cell loss in early behavioral variant frontotemporal dementia. Cereb Cortex 22: 251-259, 2012.

39. Allman JM, Watson KK, Tetreault NA and Hakeem AY: Intuition and autism: A possible role for Von Economo neurons. Trends Cogn Sci 9: 367-373, 2005.

40. Moretti R, Torre P, Antonello RM, Cazzato G and Bava A: Frontotemporal dementia: Paroxetine as a possible treatment of behavior symptoms. A randomized, controlled, open 14-month study. Eur Neurol 49: 13-19, 2003.

41. Lebert F, Stekke W, Hasenbroekx C and Pasquier F: Frontotemporal dementia: A randomised, controlled trial with trazodone. Dement Geriatr Cogn Disord 17: 355-359, 2004.

42. Kril JJ, Macdonald V, Patel S, Png F and Halliday GM. Distribution of brain atrophy in behavioral variant frontotemporal dementia. J Neurol Sci 232: 83-90, 2005.

43. Taniguchi S, McDonagh AM, Pickering-Brown SM, Umeda Y, Iwatsubo T, Hasegawa M and Mann DM: The neuropathology of frontotemporal lobar degeneration with respect to the cytological and biochemical characteristics of tau protein. Neuropathol Appl Neurobiol 30: 1-18, 2004.

44. Wei H, Dobkin C, Sheikh AM, Malik M, Brown WT and Li X: The therapeutic effect of memantine through the stimulation of synapse formation and dendritic spine maturation in autism and fragile X syndrome. PLoS One 7: e36981, 2012. 